\title{
List of reviewers
}

JOURNAL OF ADVANCES IN CHEMISTRY (2017).

The editorial team of the journal would like to thank the following reviewers for their work in referring manuscripts during 2017.

Manimaran Rajavelu

Deivasigamani

Agilan C. V.

Mahmoud Shaker Hussain

Pradeep Gurunathan

M.G. Kavitha

Parvin Kumar

A. Srithar

Ravichandran M

Kirubakaran Balakrishnan

M.Baritha Begum Begum

Malathi D

Siham Lhimr

Palash Dutta

Yelda Hangun Balkir

Mouayed Yousif Kadhum

Mohana Prabha

Mahmood Ali Hasan

C.M. Narayanan

Palanivel Rajan

A. Narendra Kumar

Anamitra Chatterjee

Sampath Krishnan

Anandakrishnan V

Sankar Ganesh R

S. Rajesh

S.M. Mazhar Nazeeb Khan

Ahmed H. Osman

I. Balasubramanian

Praynlin Edinson

S. Gopinath
Pandi. K

Theshini Perera

B. Govindh

Vijay Anandh

Deepkumar Joshi

Prince Sahaya Sudherson

Swapnila Roy

Aida Chaker

Krishnaraj S

Hitesh Chopra

Kanthavelkumaran Natesan

S. Karthikeyan

Pradeep Thirumoorthy

Ashraf Aly

Marcia Eugenia Ojeda Morales

S. Magibalan

Padmalaya G

Loganathan Shanmugam

A. Mohankumar

Ashraf M Mahmoud

S. Abdelnabi

Alaa M. Hayallah

Siva Ness Kumar

Dr. Afsanehsadat Larimi

A.S. Anakath

Kulwinder Dhindsa

Sayyed Hussain

Hussain J. Mohammed

Saima Imad

Guliyev Nihad Gudret
Banumathi Sembanan

Rajeswari Balakrishnan

Vijay Kumar R

Chen Ya Ling

Shridhar N Mathad

Padmanaban M S

Vaira Prakash

Dinesh Kumar Myilsamy

T. Manoharan

Magda.H. Abdellattif

Liam Critchley

Siva Kumar

N. Saravanan

Swaminathen Swaminathen

Swaminathan

Ankur Singh Bist

D. Maharajan

Nodirali Normakhamatov

Raj Mukherjee

Karthikeyan. G

Yasser El-Nahhal

Rajat Subhra Das

Bendjeddou Amel

Sambhani Naga Gayatri

Sheeja Ku Mari

V Sivaramakrishnan

M Varatharajulu

Vittel Rao R K

D.Sivananda Kumar

Imad Osman Abu Reid 